written materials may not be the optimum medium for this, particularly with the rise in remote consultations following the Covid-19 pandemic. Mobile applications (Apps) have shown early promise as patient education tools. In particular, 'Avatar'-based Apps, where a virtual, interactive 3D character serves as a teacher, have shown potential in other conditions such as ischaemic heart disease and rheumatoid arthritis.

Methods A moderated, structured focus group of $8 \mathrm{HF}$ patients and one patient carer was held to explore their experiences with patient education and identify areas in which HF education and knowledge of self-care was lacking. All participants shared their thoughts during moderated discussion, and recurring themes were identified. Participants then developed a 'curriculum' of topics that they considered most important in an educational App, with input from the HF team.

Results Key themes that emerged from patients' prior experience of patient education included feelings of being overwhelmed and isolated during the initial diagnosis of HF, the importance of a trusted medium for accessing health information (and fear of misinformation), and 'a picture says a thousand words' - visual information was easier to absorb than text. The educational curriculum developed by participants prioritised the following areas: impact on lifestyle, medical management (including the importance of HF medicines), lifestyle changes and care planning, and self-management (including monitoring for signs of deterioration). A prototype App was developed in conjunction with Cognitant Group, using an Avatar (figure 1). The Avatar was designed to be a patient expert in $\mathrm{HF}$, aged $\sim 65$ years old. A living room setting was picked for the content for viewers to feel more relaxed. For Avatar movements to seem natural, voice and movements were recorded and synchronised using motion-capture technology. The first module entitled 'Living with heart failure' outlines of the following topics: explaining $\mathrm{HF}$ and the typical patient journey, the purpose of HF medications, lifestyle advice (including exercise and healthy eating, smoking and alcohol, sex and relationships), self-monitoring of HF symptoms, when to call for help and signposts to trusted HF educational resources. Acceptability of the App is currently being evaluated in the HF cohort at this specialist centre.

Conclusions Avatar-based Apps may be a useful tool in patient education in HF when co-designed with patients. Further research will evaluate the acceptability of such an App to a cohort of patients in a specialist HF centre.

Conflict of Interest Laboratoires Servier provided funding for the development of the App. Dr Singhal's salary is funded by a fellowship from Abbott.

\section{ONE YEAR OUTCOMES OF HEART FAILURE MULTISPECIALTY MULTIDISCIPLINARY TEAM VIRTUAL MEETINGS}

${ }^{1}$ Hani Essa, ${ }^{1}$ Emeka Oguguo, ${ }^{1}$ Homeyra Douglas, ${ }^{1}$ Alice Foster, ${ }^{1}$ Lauren Walker, ${ }^{1}$ Justine Hadcroft, ${ }^{1}$ Julie Bellieu, ${ }^{2}$ Matthew Kahn, ${ }^{2}$ Archana Rao, ${ }^{1}$ Dan Cuthbertson, ${ }^{1}$ Asan Akpan, ${ }^{1}$ Christopher Wong, 'Rajiv Sankaranarayanan. 'Liverpool University Hospital, Liverpool, UK; ${ }^{2}$ Liverpool Heart and Chest Hospital

10.1136/heartjnl-2021-BCS. 129

Background/Introduction Heart Failure is frequently associated with several comorbidities such as ischaemic heard disease, diabetes mellitus, chronic obstructive pulmonary disease, chronic kidney disease and frailty. This level of complexity is best dealt with by a multispecialty multidisciplinary team (MDT) model.

Methods This was a single centre observational study (January 2020-December 2020) that was undertaken in a British university hospital looking at effect of HF multispecialty virtual MDT meetings on HF outcomes. Patients acted as their own controls outcomes compared for equal period pre versus post MDT meeting. The multi-specialty meeting was conducted once monthly via video-conferencing. It consisted of heart failure cardiologists (from primary secondary and tertiary care), heart failure specialist nurses (hospital and community), nephrologist, endocrinologist, palliative care specialists, chest physician, pharmacist, pharmacologist and geriatrician. Recommendations were made as consensus from the multispecialty meeting. The main outcome measures were 1) number of hospitalisations and 2) outpatient clinic attendances 3) cost savings.

Results A total of 189 patients were discussed from JanuaryDecember 2020. This was uninterrupted during the COVID19 pandemic. The mean age was $70.3 \pm 18.1$ years and median follow-up 6 months (range 1-13 months). The mean Charlson Co-morbidity score was $5.3 \pm 1.2$ and Rockwood Frailty Score was $4.9 \pm 1$. The mean number of outpatient clinic attendances avoided was $1.7 \pm 0.4$. This reduced inconvenience to patients, saved patients money (transport and parking costs) and led to carbon footprint reduction. The MDT meeting total costs were $£ 15,400$ and the 31 clinic appointments they generated cost an estimated £3720. However, the MDT meetings prevented 277 clinic appointments (cost saving $£ 33,352$ ). Finally, the mean number of hospitalisations pre-MDT was 0.7 Vs 0.2 post MDT $(\mathrm{p}<0.01)$ with a saving of around 730 bed days (estimated cost-saving $£ 260,000)$.

Conclusion The HF multispecialty virtual MDT approach provides seamless integration of primary care community services with secondary and tertiary care. Consensus decision from MDT meetings provides holistic approach for $\mathrm{HF}$ patients with comorbidities and frailty, and reduces inconvenience to patients by preventing the need to attend multiple specialty clinics. This approach can also lead to significant cost-savings to the healthcare system.

Conflict of Interest nil

\section{DEVELOPMENT OF OUTPATIENT BASED ACUTE HEART FAILURE CARE CALLS FOR DEVELOPMENT OF CLINICAL PSYCHOLOGY SERVICE FOR WHOLE-PERSON CARE PROVISION IN THE UK}

${ }^{1}$ Abdullah Abdullah, ${ }^{2}$ Suzanne Wong, ${ }^{3} J o h n$ Cleland, ${ }^{4}$ Kenneth Wong. ${ }^{1}$ Blackpool Victoria Hospital NHS Foundation Trust Blackpool, Blackpool, UK; ${ }^{2}$ Blackpool Victoria Hospital; ${ }^{3}$ University of Glasgow, UK; ${ }^{4}$ Blackpool Teaching Hospitals

\subsection{6/heartjnl-2021-BCS.130}

Introduction Acute heart failure (AHF) is associated with 7 $11 \%$ mortality and substantial morbidity. Whole person care of patients with HF is important as depression and hopelessness are prevalent. Patients may feel more hope if they can be successfully treated with IV diuretics at home, in the community or hospital ambulatory care [outpatient management (OPM)]. In 2016, 24\% of HF services offered OPM in the 